\title{
The essential necessity to invest in the cultivation of peer recovery specialists as organizational leaders
}

\author{
Traci Carrano Jones \\ The Chicago School of Professional Psychology, Washington, District of Columbia, \\ USA, and \\ Darrell Norman Burrell \\ Florida Institute of Technology, Melbourne, Florida, USA
}

\begin{abstract}
Purpose - The emergence of COVID-19 has exacerbated and spurred the growth of mental health issues in ways that have challenged mental health workers tremendously. The complex nature of COVID-19 has made the need of mental health professionals extremely important. Hospital ward overcrowding, social distancing requirements, sequestrations, limits to face-to-face consultations have created barriers to mental health access, especially those in need of Certified Peer Recovery Specialists. Certified Peer Recovery Specialists play a critical role in the treatment of mental illness through their support and engagement of those recovering from substance abuse. Many in this role are hired because they have a strong skillset for help-oriented clinical work but are often challenged, especially in times like this to development and demonstrate leadership skills. This paper explores the nature and need to develop leadership skills and leadership challenges for people in this field through interpretative phenomenological analysis (IPA) and a content analysis of the current and seminal literature.

Design/methodology/approach - This paper explores the nature and need to develop leadership skills and leadership challenges for people in mental health through IPA and a content analysis of the current and seminal literature.

Findings - The essential necessity to invest in the cultivation of peer recovery specialists and mental health professionals as organizational leaders.

Originality/value - Hospital ward overcrowding, social distancing requirements, sequestrations, limits to face- to- face consultations have created barriers to mental health access, especially those in need of Certified Peer Recovery Specialists. Certified Peer Recovery Specialists play a critical role in the treatment of mental illness through their support and engagement of those recovering from substance abuse.
\end{abstract}

Keywords Peer-to-peer networks, Peer recovery specialist, Mental health managers, Addiction leadership, Counseling leadership, Health coaching

Paper type Research paper

\section{Introduction}

Mental health professionals are charged with caring for people with lived experiences with mental health and substance use struggles (Greenberg, 2020; Kar and Singh, 2020). One demographic of professionals who have become a fixture in the mental health workplace is the peer provider or peer recovery, specialist. These individuals use their own lived experiences coupled with training, supervision and certification to share their hope with

(C) Traci Carrano Jones and Darrell Norman Burrell. Published in PSU Research Review. Published by Emerald Publishing Limited. This article is published under the Creative Commons Attribution (CC BY 4.0) licence. Anyone may reproduce, distribute, translate and create derivative works of this article (for both commercial and non-commercial purposes), subject to full attribution to the original publication and authors. The full terms of this licence may be seen at http://creativecommons.org/licences/by/4.0/ legalcode.

Cultivation of peer recovery specialists

Received 7 January 2021 Revised 26 May 2021 6 January 2022

Accepted 8 January 2022

Accep 
others to help them along their journeys toward recovery. Their leadership, however, is not without challenges. Inherent in our society is the stigma associated with having a mental illness, regardless of the qualifications earned to perform a job adequately and effectively (Greenberg, 2020; Kar and Singh, 2020). As a result, the development of innovative and collaborative leadership skills has never been more critical (Greenberg, 2020; Kar and Singh, 2020). There exists a very limited amount of peer-reviewed literature concerning peer recovery specialists. As a result, this topic needs further exploration, and this paper attempts to engage in this topic and break new ground in the literature by representing the first study of its kind on the occupation of peer recovery specialists.

The old paradigm of leadership, originating with theories of the 18th century (Rost, 1995), involved an authoritarian style of leading by invoking power over others, working within groups' frameworks and focusing on goal achievement (Melnic, 2009).

According to Melnic (2009), authoritarian leaders engage from the viewpoint the employees need to be overmanaged; otherwise, they will not do the work and have no interest in working to the best of their ability. Over time, leadership theorists have designed models that have shifted the process from managing employees to leading employees (House et al., 2002). Just like leadership and management functions have evolved, so has the critical importance of leadership in mental health fields.

The World Health Organization (WHO, 2019) reported that work is good for mental health. Peer recovery specialists are no different. These professionals have lived experiences like those they aim to help, coupled with training, supervision and certification (Ahmed et al., 2015). Peer recovery specialists are associated with a plethora of positive outcomes such as improvements in service recipients' functioning, housing stability, quality of life, satisfaction with care and personal recovery and reducing the number of hospitalization days and use of crisis services (Mancini, 2018). Despite peer recovery specialists being positioned to make a big difference in the lives of people with mental health challenges, the system does not adequately support them. When peer recovery specialists do not receive full backing from institutions, policies and decisionmakers, the whole mental health community suffers because the peer specialist is demoralized. Those who might have benefited from peer support do not receive the services they could have. On another level, because the peer recovery specialist is also part of the mental health community as a service recipient, the damage done is compounded. The peer recovery specialist is demoralized as a professional in their field. Because they are part of the mental health community, they are also discouraged from their community.

Zeng et al. (2020) posited a list of challenges experienced by peer mental health providers (known by various names, such as peer recovery specialists, recovery coaches, peer support workers, peer counselors, etc.), including failing to establish professional credibility of the peer provider, not operationally defining the roles of peer providers, the continuing stigmatizing attitudes of professional mental health colleagues, the collaboration and integration of interdisciplinary teams lack support for peer providers. There are inadequate social and mental health policies for peer providers. Ahmed et al. (2015) contributed a list of other challenges experienced by peer mental health providers such as challenging work conditions, inadequate compensation, insufficient training, the taxing emotional costs of supporting peers and the complex nature of the triple identity of being a provider, peer and consumer.

\section{Professional credibility}

Zeng et al. (2020) listed failing to establish the peer provider's professional credibility as one of the significant challenges experienced by this mental health professional. Delman and Klodnick (2017) advised that mutual sharing with the clients, a basic tenet in peer support work, is viewed as unprofessional and contradictory by other clinical staff. Moran et al. (2013) 
agreed that peer providers should not share so much of themselves. Moran et al. (2013) asserted that there is a lack of appreciation of the peer providers' roles by others and that the industry's lack of professional standards gives peer providers "a bad name." Gate and Akabas (2007) asserted that this failure to operationalize the peer provider or implement a recovery mindset renders indifference and hostility toward the peer providers. In support of this notion, Vandewalle et al. (2016) reported that some nurses in clinical settings develop patronizing attitudes and beliefs about peer providers. Peer providers are not taken as seriously as other workers, not by other staff or clients (Moran et al., 2013). Scholz et al. (2017) suggested that mental healthcare's traditional hierarchical nature places the power difference in the stark contrast between providers and peers, making it so peer providers are not indeed recognized. Many mental health practitioners harbor concerns about consumers holding leadership positions due to skill level concerns or concerns about the relapse of mental health symptomology (Scholz et al., 2017). Peer providers experience this lack of credibility firsthand. Peer providers perceive that they are not accepted by stakeholders of the organizations they are affiliated with because of this lack of credibility (Vandewalle et al., 2016). Besides, peer providers feel they must always justify their position and qualifications due to a lack of credibility exacerbated by professional standards and inadequate training and compensation. Vandewalle et al. (2016) noted that the substandard pay and nonstandardized certification deplete the credibility and prospects for funding and risk professional sustainability.

\section{Roles of peer providers}

A second challenge identified by Zeng et al. (2020) was the unclear definition of a peer provider's role. One of the key elements of peer providers is that they share their lived experiences with others so that their experiences, strength and hope may help others going through something similar. Delman and Klodnick (2017) maintained that this premise of sharing personal stories by professionals to clients challenges a professional paradigm that emphasizes boundaries, and this practice is considered potentially harmful. Moran et al. (2013) cited many elements related to challenges related to the peer provider's roles, that is, role confusion, conflicts with service recipients, dual relationships, role conflicts and working where one receives (or received) services. Job descriptions are also ill-defined in many locations. Some locations use peer providers for case management, group facilitation, transportation or medication dispensing (Moran et al., 2013). Many researchers argued that there is role confusion for peer providers (Gates and Akabas, 2007; Scholtz et al., 2017; Vandewalle et al., 2016), leading to frustration. This confusion can manifest as being both provider and recipient of services, being both friend and fellow consumer or being both provider and coworker (Vandewalle et al., 2016).

\section{Stigma}

Stigmatizing attitudes toward peer providers constitutes a third challenge identified by Zeng et al. (2020). Although faced with stigma by their nonpeer colleagues, young adult peer providers seem to navigate more effectively when they have more psychological capital (i.e. self-efficacy, optimism, perseverance, hopefulness and resilience) (Delman and Klodnick, 2017). The stigmatizing attitudes were evident by the negative comments about clients made by the nonpeer mental health professionals outside of appointment times and negative behavior directed toward the peer providers by their nonpeer colleagues due to their diagnosis (Moran et al., 2013; Vandewalle et al., 2016). The attitude and belief by nonpeer staff continue that recovery is not genuinely possible for the peer provider; thus, stigma ensues (Gates and Akabas, 2007). Vandewalle et al. (2016) contend that peer workers have a perception that their nonpeer counterparts' prejudice of peer workers that they cannot cope

Cultivation of peer recovery specialists

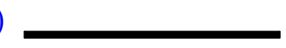


when they experience work-related stress is counterproductive. Mental health struggles bring with them a mark that signifies a stigma within our society. Mental health practitioners are charged with a task to help their clients overcome this societal stigma and the self-stigma that accompanies it. Corrigan (2018) describes the unintended consequences of the standard mental health campaigns, which help perpetuate stigma. Corrigan (2018) suggests that mental health practitioners need to empower peers instead of "fixing" them.

\section{Collaboration and integration of interdisciplinary teams}

Another area identified by Zeng et al. (2020) that is challenging for peer providers in the workplace concerns collaboration and integration of interdisciplinary teams. Through a capital theory lens, Delman and Klodnick (2017) identified eight factors that seemed to signify on-the-job success: persistence, job confidence, resilience, job training, professional communication with colleagues, regular and individualized supervision, support from colleagues and family support. These findings suggest support for refining social, organizational capital and increasing individualized cultural, social and psychological capital via training and supervision. Moran et al. (2013) asserted that the implementation of peer workforce clinical case management teams is challenging. Happell and Roper (cited in Scholtz et al., 2017) described consumer leadership as involving managing, planning, funding or researching. These leaders can make important decisions and help manage resources. It is imperative to avoid tokenistic practices, which can be accomplished by partnering with consumer leaders to set the organization's agendas (Scholtz et al., 2017). There are discrepancies between peer workers and mental health professionals about the right course of action for service users. Peer workers feel isolated and not as equal team members (Vandewalle et al., 2016). Peer workers are neither service user nor professional in this scenario. This could be linked to the undefined role and unrealistic responsibilities (Vandewalle et al., 2016). Edmondson (2019) described "teaming," which requires that nonpeers and peer workers work together in a psychologically safe way. Psychological safety ensures that opinions are respected on all sides, where peers and nonpeers can help the service recipients.

\section{Social and mental health policies}

Zeng et al. (2020) concluded their assessment of peer providers' challenges in the workplace by adding social and mental health policies to the mix. Delman and Klodnick (2017) added that while most states require intensive training and a written exam to become certified, this is not standardized across the profession. The process focuses on professional, ethical standards and specific recovery-focused approaches to engage clients. Moran et al. (2013) requested a standardized development of manuals and models for peer providers' professional development. They also identified a need for job placement and supervision of paid peer providers. The authors cited a lack of uniformity of peer training, work structure problems and low pay as other challenges. Scholz et al. (2017) asserted a need for peers' involvement in planning, implementing and delivering policy. They maintained that the overall goal is to improve mental health service delivery in government, private and community sectors. The value added by peers cocreating in an organization by paying attention to consumers' values and the organization's values increases the organization's value overall. By allowing the peers to determine what training and development they need, the organization only benefits (Scholtz et al., 2017). Some peers fear standardized training will make them "professional," and they will lose that "peer" quality (Vandewalle et al., 2016). Gates and Akabas (2007) maintained that the rules about confidentiality need more clarity, and there need to be more social networking opportunities. Recover models emphasize social inclusion (Vandewalle et al., 2016), supported by social networking. Self-determination places 
value on managing mental health. Thus, to maintain a recovery focus, implementing lived experiences through peer providers and mutual support at all levels of the organization is imperative (Vandewalle et al., 2016). Vandewalle et al. (2016) did identify a need to have mental health policies for peer providers to aid in their dealings with service users in crisis or with self-injury behavior.

\section{Appreciative leadership}

Whitney et al. (2010) delivered a conceptual way for leaders to address difficulties they encounter within their organizations. Discontent between nonpeer colleagues and peer recovery specialists would be one of these difficulties. The basic tenets of this model are Inquiry, Illumination, Inclusion, Inspiration and Integrity.

Inquiry challenges leaders to ask their employees to share their thoughts and feelings. These leaders seek to share stories of success or ideas for the future. These behaviors encourage people and let them know that they are valued and so are their contributions. Whitney et al. (2010) advice leaders to ask questions and truly listen to what peer recovery specialists have to say. What might this look like would be to ask what they have learned in their training to be a peer recovery specialist? What are how they believe they can contribute to the organization? What experiences have they had in their lives that have well prepared them to perform in these capacities? What areas do they feel they might need support, and what would that support look like? By asking these positive questions, the peer recovery specialist will feel listened to and feel empowered. They will also likely listen to others in return and be more likely to follow the leaders in charge of less disgruntled. The environment for all will be a little more harmonious.

The second topic in Appreciative Leadership is Illumination, which helps people understand how they can best contribute. During this stage, strengths are uncovered of both the individuals and their coworkers. Confidence is gained, and encouragement is garnered for each other as everyone works through their strengths. This commences during the inquiry stage by asking questions that also address coworkers' strengths, such as: Are you curious about your colleagues' unique experiences and how these can help others? What about their skills, abilities, hopes and dreams, and how these can enrich our organization? Leaders who observe the evidence-based success of peer recovery specialists will see that it is a milieu that works. This is a second component to illumination, seeing what works when people are at their best (Whitney et al., 2010). To spread the word of peer recovery specialists' aptitude, leaders need success stories of best practices for learning and standardization (Whitney et al., 2010). This third illumination recognizes peer recovery specialists for their accomplishments and breaks down stigma. Finally, Whitney et al. (2010) suggest aligning strengths for development and collaborative advantage. To do this, Whitney et al. (2010) advocate providing opportunities for the peer recovery specialist to do what they do well while collaborating with others whose strengths are complementary, perhaps their nonpeer colleagues. This cohesion in practice will be the best for all involved.

Inclusion is next and is perhaps self-explanatory. Collaboration is critical as a sense of belonging, and cocreation is practiced. Being a part of something in an environment and a culture conducive to this concept can be very productive. This tenet involves the stakeholders of the community. To be inclusive constitutes being inclusive of everyone who might be affected. Although the list is not exhaustive, this list might include peer recovery specialists, clinical practitioners, supervisors, researchers, service recipients, executives, policymakers, Board of Directors, other frontline staff, managers, suppliers, shareholders, foundations, advocacy groups, family members, think tanks, artists, futurists, community members and mental health watch groups. Everyone on this list should be invited to the table for conversation and input. By doing this, peer recovery specialists are taken seriously and are
Cultivation of peer recovery specialists 
not secreted away. This encourages diversity, and inclusion makes it safe to speak up (Whitney et al., 2010).

A sense of direction is delivered from the next step, Inspiration (Whitney et al., 2010). A leadership direction is developed, which can bring about hope and release energy. If a leader recognizes the peer recovery specialists' ability to relate to the service recipients and inspire hope, then, in turn, the organization can inspire hope, dreams and vision. Harnessing the peer recovery specialists' positive power of inspiration can transpire to other support team members and benefit the whole organization. Besides, by recognizing the motivation within the peer recovery specialist, the leader is, in effect, inspiring the leadership potential of the peer recovery specialist. Inspiration can be the foundation for transformation, innovation and sustainable high performance (Whitney et al., 2010).

Finally, integrity sets the example by which the organization will be known. Integrity can be recognized when the organization displays strong moral principles. The leaders and the employees, both nonpeers and peer recovery specialists, will be expected to deliver their best for the greater good and establish trust and dependability on each other. A leader in a supervisory role of peer recovery specialists needs to have integrity. This constitutes working toward wholeness and supporting and enabling others to do the same (Whitney et al., 2010). Whitney et al. (2010) define this on multiple levels: "seeking the whole story, aligning the whole organization, caring for the whole world, applying holistic approaches, and being open to the "holy" (p. 161). By this definition, it is evident that peer recovery specialists are part of the "whole" and should be treated accordingly. Thus, the credibility of their professional titles should be recognized. Another aspect of integrity is conscious decision-making, whereby the supervisor is charged with making decisions based on his or her values. In doing so, the supervisor can garner respect through communication and transparency while honoring the peer recovery specialists' dignity. This empowers the peer recovery specialist to draw upon their principled performance strengths (Whitley et al., 2010). Together, these tenets give hope for peer recovery specialists and their nonpeer colleagues.

\section{Transforming leadership for mental health professionals}

The notion of "transforming leadership" is derived from the formative research of Burns (1978). Burns described transforming leadership in conditions of mundane (i.e. transactional) leaders and exceptional (i.e. transforming) leaders, with the two leaders functioning at divergent ends of the leadership continuum. Burns characterized transforming leadership as behaviors and actions focused on leveraging employees' intellectual capital, expertise and experiences. According to Bass $(1985,1990,1997,1999)$, transformational leaders develop their followers' work interests and awareness and promote acceptance of the organization's purpose and mission (Bass, 1990; Bass and Avolio, 1993). Research of the transformational leadership traits among healthcare managers has reinforced the conception that transformational leadership fosters a healthy organizational culture and underwrites organizational effectiveness (Greenburg, 2020; Corrigan et al., 2002) and affords constructive influences for workers and stakeholders (Yang, 2009). Transformational leadership extends a distinctive benefit to those working in mental health job roles. Transformational leaders concentrate their activities and resources on innovation, empowerment and employee encouragement at all levels (Gumusluoglu and Illsev, 2009). Gumusluoglu and Illsev (2009) indicated that mental health workers needed to be resourceful problem-solvers because their clients have complicated, inconsistent and evolving service and support needs. Besides, Davidovitz et al. (2007) claimed that influential leaders were reflective, attuned and responsive to followers' needs, provide coaching and nurture creativity, initiative and independence among their employees and stakeholders.

According to Gumusluoglu and Ilsev (2009), transformational leaders have elevated performance expectations of their workers just as mental health professionals have 
heightened clients' expectations. Furthermore, Gumusluoglu and Ilsev (2009) expounded that transformational leaders persuade their employees to transform their personal values, selfconcept, needs and ambitions to an elevated level, which resemblances how successfully mental health and peer recovery specialists inspire their clients. Newer management concepts have evolved to focus on collaboration, cooperation and teamwork (Bass and Avolio, 1993). One crucial archetype of leadership embraces the role of transformational leadership in engaging employees in ways that inspire, motivate and empower them to a higher sense of self-value (Bass, 1985, 1990, 1997, 1999). Additionally, Bass (1997, 1999) explained that transformational leaders, through their behaviors, can constructively change and improve organizational culture.

Elpers and Westhuis (2008) contended that effective management and leadership are about investment in workers' development and creating workplaces that are focused on learning and development. According to Davidovitz et al. (2007), transformational leaders develop followers' sense of worth, allow them to explore new challenges, support their development of new skills, affirm their ability to handle challenges and support their individual growth.

\section{Personality traits of transformational leaders}

Researchers have found specific personality traits commonly associated with transformational leaders. When transformational leaders act as role models, their followers are viewed as a person willing to sacrifice their interests for the collective good and bolstering a sense of group belongingness and cohesion (Wang et al., 2011).

Bass (1985) asserted that transformational leaders support their followers when they challenge existing assumptions and provide new ideas. According to Wang et al. (2011), when transformational leaders allow their followers to offer new ideas and implement their ideas, they reassure their followers that such behavior is encouraged and do not punish their followers for failing to achieve the agreed-upon goal with the new concept. Instead of punishing followers for failure, transformational leaders motivate followers to learn from the failure. Lastly, transformational leaders address the needs of their followers (Bass, 1985). Bass (1997) stated that when using the key behaviors collectively, transformational leaders motivate individuals to achieve a higher performance level.

Transformational leaders encourage their staff to exceed their fundamental job duties by linking their employees' work roles to tasks they enjoy and can thrive at, causing followers to view their work as more meaningful (Wang et al., 2011). Wang et al. (2011) reported that transformational leaders encouraged their followers to achieve their set goals, increasing selfefficacy levels. Transformational leaders act as coaches and mentors by providing their followers with the support and tools they need to accomplish their jobs (Bass, 1999). According to Wang et al. (2011), transformation leaders motivate followers to work for the group's good by increasing social identification, thereby inspiring followers to engage in altruistic behaviors and dedicate themselves to their jobs and organization.

\section{Psychological safety}

Edmondson (2019) proposed an environment in an organization where learning, innovation and growth are encouraged and brought to fruition with psychological safety. This can be extended for peer recovery specialists. Psychological safety within an organization can be defined as having a climate where people, peer recovery specialists, feel safe enough to speak up and share questions, concerns and ideas (Edmondson, 2019). When peer recovery specialists are not recognized as the professionals they are, they will not feel comfortable speaking up. Edmondson (2019) posited that when people in an organization do not speak up, the organization's ability to innovate and grow is stagnated. Besides, psychological safety produces high performance and encourages the engagement of employees (Edmondson, 2019). When there is no psychological 
safety, there is a misconception of success that leads to severe business failure (Edmondson, 2019). Being completely reliant on authority can be detrimental to psychological safety (Edmondson, 2019). However, leaders who are more apt to say, "I do not know," and who value employees, including peer recovery specialists, get employees who are engaged, problem-solvers and high performers (Edmondson, 2019). Therefore, it is in the organization's best interest to establish psychological safety for peer recovery specialists.

\section{Data collection}

The aim of interpretative phenomenological analysis (IPA) is to understand how participants are making sense, experience and navigate the personal or professional worlds (Smith and Eatough, 2006). The richness of an IPA study is the direct observations and participants' lived experiences that function as the subject matter expert witnesses on an occurrence or environment (Smith and Eatough, 2006).

There were ten participants, which is acceptable for this study (Smith and Eatough, 2006). The participant group included seven females and three males. The respondents all reported to have had some college, four reporting two years of college, two reported at least one associate degree, three reported bachelor's degrees and one reported some graduate-level education. All participants were certified peer recovery specialists. The average years of experience were significantly varied from 1.5 years to 14 years. The participants were all trained professionals that were recruited through a peer recovery professional group in the state of Virginia in the USA. Since there is very limited research on this population and this field, this opportunity to collect these data could provide critical insight to those looking to improve the field and advance the profession.

\section{Data collection questions and results}

Based on your experience and the nature of the job, what do you see as the essential leadership traits required to do a peer recovery specialist's job?

(1) Competent or professional expertise in the field: Meaning that they need to understand rules, tools, steps and aspects of the job and vocation of the peer recovery specialist.

(2) Communicative: Meaning providing coaching, constructive feedback, honest communication and consistent communication.

(3) Resourceful: Meaning the ability to be innovative, creative and flexible in the way to manage limited resources.

(4) Democratic or participative or collaborative: Meaning the ability to cooperate and join forces with others so that ideas and perspectives are solicited when decisions are made.

(5) Emotional intelligence: Meaning the ability to be calm, reasonable, rational, reflective and strategic in how you interact or engage others.

(6) Compassionate: Meaning being caring, nurturing and showing empathy and sympathy in your interactions with staff and clients.

(7) Fair: Meaning treating people in ways that are equitable, impartial, unbiased.

(8) Respectful: Meaning showing respect for those of various backgrounds and perspectives. 
Based on your experience, what do you see as the value of leadership training for peer recovery specialist?

Participant 1 said, "Employers should invest more professional development in leadership training. Currently, most of the training is more clinical around the job but not around being a leader. Leadership training is important for those in the field. Each of us in the field is a source of energy, adding all the energies present in a group that includes your family, friends, colleagues, and work teams. All these socialization and interactions produce a whole set of emotions, feelings, experiences, abilities, talents, skills, and preferences that must be relentlessly well-managed to safeguard your well-being and decision making. To do this for yourself and to help clients in doing this, you must develop superior leadership skills, which makes formal leadership training very important."

Participant 2 said, "We need leadership training. There is so much limited investment in our development beyond what is required of the job. Without leadership training, more professional development options, we are treated like janitors in operation sweeping up everything. We have more value."

Participant 3 said, "The more training, the more tools in our toolbox, the more validation we have, the more knowledge we have, the more serious our roles are."

Participant 4 said, "We are treated horribly in this role. We are underpaid and underdeveloped. We are not appreciated and taken seriously."

Participant 4 reported, "Recovery is not an easy feat. I had bouts of Imposter Syndrome. I did not feel as if I had the experience to help. I doubted my abilities. I needed more leadership skills so I could feel more comfortable."

Participant 5 stated, "The job of a peer recovery specialist has high turnover because organizations are not interested in investing in their employees. High turnover and limited training hurt the quality of service to clients. How can an organization expect me to act and function like a leader when I have never been trained to do so?"

Participant 6 stated, "My job requires me to lead relationships and lead recovery efforts. As a result, leadership training should be a core aspect of the training for the job. This kind of training should be required for all professionals in the field. We need more help and more access to more learning tools to do our jobs better."

Participant 7 said, "I have never been formally offered any leadership training. What I know about leadership and leading people, I learned from other jobs, and yet the job requires that I lead people on a path to recovery when I have never been formally trained in leadership. I see a huge issue with that. To do our jobs better, we need more training and more resources from our organizations for professional development."

Participant 8 said, "You have to be a great leader to do this job effectively. The nature of the job is ethical leadership, leadership decision-making, Servant leadership, and compassionate leadership. I will challenge you that someone that does not have a superior orientation in those areas is not the most effective person in this job. The training that we need is deficient."

Participant 9 stated, "You have to have leadership skills to meet the challenges of the job, which is human enhancement. Peer recovery specialists must lead the employment of processes inherent to developing personal potential with a view of strengthening and making its integral evolution more effective. Anyone in this role must coordinate individualized education and developmental plans. You need to have the ability to ask intelligent questions and listen attentively when working with clients. Your problem-solving skills need to be sharp enough to handle crises and set good examples. The ability to coordinate and manage these efforts and activities requires strong leadership and organizational skills."

Participant 10 said, "Next to the clinical skills required to do the job, leadership training is critical. Why? Because we are leading patients, leading programs, leading projects, and leading treatment plans. The training we get to do our jobs, in general, is not sufficient." 
Zeng et al. (2020) proposed a list of leadership challenges experienced by peer recovery specialists, some of which were echoed in the interviewees' responses. For instance, Zeng et al. (2020) cited failing to establish the peer provider's professional credibility as one of the challenges. At least one of the interviewees was noted as stating that their roles were not always taken seriously. Another challenge reported by Zeng et al. (2020) was that the peer recovery specialist's role is not uniformly operationally defined. The continuing stigmatizing attitudes of professional mental health colleagues is another challenge noted by Zeng et al. (2020). Ahmed et al. (2015) also contributed a list of challenges experienced by peer recovery specialists. Among these included insufficient training. All ten respondents endorsed this theme. Some responses argued that the lack of training and "CEUs" led to inadequate job availability. The complex nature of the triple identity of being a provider, peer and consumer was another challenge proposed by Ahmed et al. (2015).

These results shed light on the need for more studies and research on a peer recovery specialist's leadership development and job roles. The results indicate a need to train professionals in mental health and those in peer recovery beyond clinical duties and leadership. Formal leadership training should be required for employees at every level in mental health and those in peer recovery roles.

\section{Recommendations for future research}

Further research exploring the effect of leadership on staff retention in mental healthcare should be examined further. There should be further research that explores what does job satisfaction looks like for mental health and peer recovery specialists. There should be further research exploring the ideal work culture for mental health and peer recovery specialists.

\section{References}

Ahmed, A., Hunter, K., Mabe, A., Tucker, S. and Buckley, P. (2015), “The professional experiences of peer specialists in the Georgia mental health consumer network", Community, Vol. 51, pp. 424-436.

Bass, B.M. (1985), Leadership and Performance beyond Expectations, Free Press, New York, NY.

Bass, B. (1990), "From transactional to transformational leadership: learning to shwas the vision", Organizational Dynamics, Vol. 18 No. 3, pp. 19-31, doi: 10.1016/0090-2616(90)90061-S.

Bass, B. (1997), "Does the transactional-transformational leadership paradigm transcend organizational and national boundaries?", American Psychologist, Vol. 52 No. 2, pp. 130-139.

Bass, B. (1999), "Two decades of research and development in transformational leadership", European Journal of Work and Organizational Psychology, Vol. 8 No. 1, pp. 9-32.

Bass, B.M. and Avolio, B.J. (1993), "Transformational leadership: a response to critiques", in Chemers, M.M. and Ayman, R. (Eds), Leadership Theory and Research: Perspectives and Directions, Academic Press, San Diego, CA, pp. 3-34.

Burns, J.M. (1978), Leadership, Harper \& Row, New York, NY.

Corrigan, P. (2018), The Stigma Effect, Columbia University Press, New York.

Corrigan, P.W., Diwan, S., Campion, J. and Rashid, F. (2002), "Transformational leadership and the mental health team", Administration and Policy in Mental Health, Vol. 30 No. 2, pp. 97-109.

Davidovitz, R., Mikulincer, M., Shaver, P.R., Izsak, R. and Popper, M. (2007), "Leaders as attachment figures: leaders' attachment orientations predict leadership-related mental representation and followers' performance and mental health", Journal of Personality and Social Psychology, Vol. 93 No. 4, pp. 632-650, doi: 10.1037/0022-3514.93.4.632.

Delman, J. and Klodnick, V. (2017), "Factors supporting the employment of young adult peer providers: perspectives of peers and supervisors", Community Mental Health Journal, Vol. 53, pp. $811-822$. 
Edmondson, A. (2019), The Fearless Organization: Creating Psychological Safety in the Workplace for Learning, Innovation, and Growth, Wiley, New Jersey.

Elpers, K. and Westhuis, D.J. (2008), "Organizational leadership and its impact on social workers' job satisfaction: a national study", Administration in Social Work, Vol. 32 No. 3, pp. 26-43, doi: 10. 1080/03643100801922399.

Gates, L. and Akabas, S. (2007), "Developing strategies to integrate peer providers into the staff of mental health agencies", Administration and Policy in Mental Health and Mental Health Services Research, Vol. 34 No. 3, pp. 293-306.

Greenberg, N. (2020), "Mental health of healthcare workers in the COVID-19 era", Nature Reviews Nephrology, Vol. 16, pp. 425-426, doi: 10.1038/s41581-020-0314-5.

Gumusluoglu, L. and Ilsev, A. (2009), "Transformational leadership, creativity, and organizational innovation”, Journal of Business Research, Vol. 62 No. 4, pp. 461-473, doi: 10.1016/j.jbusres.2007. 07.032 .

House, Javidan, Hanges and Dorfman (2002), "Understanding cultures and implicit leadership theories across the globe: an introduction to project globe", Journal of World Business, Vol. 37 No. 1, pp. 3-10, doi: 10.1016/S1090-9516(01)00069-4.

Kar, S.K. and Singh, A. (2020), "Mental health of mental health professionals during COVID-19 pandemic: who cares for it?", Asian Journal of Psychiatry, Vol. 53, 102385, doi: 10.1016/j.ajp.2020.102385.

Mancini, M. (2018), "An exploration of factors that affect the implementation of peer support services in community mental health settings", Community Mental Health Journal, Vol. 54, pp. 127-137.

Melnic, A. (2009), “The leadership style as a predictor of the work styles within the group”, Economy Transdisciplinarity Cognition, Retrieved from ABI/INFORM Global, Vol. 1, pp. 59-64.

Moran, G., Russinova, Z., Gidugu, V. and Gagne, C. (2013), "Challenges experienced by paid peer providers in mental health recovery: a qualitative study", Community Mental Health Journal, Vol. 49, pp. 281-291.

Rost, J.C. (1995), "Leadership: a discussion about ethics", Business Ethics Quarterly, Vol. 5 No. 1, pp. 129-142, doi: 10.2307/3857276.

Scholz, B., Bocking, J. and Happell, B. (2017), "How do consumer leaders co-create value in mental health organizations?", Australian Health Review, Vol. 41, pp. 505-510.

Smith, J.A. and Eatough, V. (2006), "Interpretative phenomenological analysis", in Breakwell, G., Fife-Schaw, C., Hammond, S. and Smith, J.A. (Eds), Research Methods in Psychology, 3rd ed., Sage, London.

Vandewalle, J., Debyser, B., Beeckman, D., Vandecasteele, T., Van Hecke, A. and Verhaeghe, S. (2016), "Peer workers' perceptions and experiences of barriers to implementation of peer worker roles in mental health services: a literature review", International Journal of Nursing Studies, Vol. 60, pp. 234-250.

Wang, G., Oh, I.S., Courtright, S.H. and Colbert, A.E. (2011), "Transformational leadership and performance across criteria and levels: a meta-analytic review of 25 years of research", Group and Organization Management, Vol. 36 No. 2, pp. 223-270, doi: 10.1177/1059601111401017.

Whitney, D., Trosten-Bloom, A. and Rader, K. (2010), Appreciative Leadership: Focus on what Works to Drive Winning Performance and Build a Thriving Organization, McGraw, New York.

World Health Organization (WHO) (2019), "Mental health in the workplace", available at: https://www. who.int/mental_health/in_the_workplace/en/.

Yang, Y. (2009), "An investigation of group interaction functioning stimulated by the transformational leadership on employee intrinsic and extrinsic job satisfaction: an extension of the resourcebased theory perspective", Social Behavior and Personality: An International Journal, Vol. 37 No. 9, pp. 1259-1277.

Zeng, G., Chung, D. and McNamara, B. (2020), "Organisational contexts and practice developments in mental health peer provision in Western Australia", Journal of Health Organization and Management, Vol. 34 No. 5, pp. 569-585.
Cultivation of peer recovery specialists 


\section{Further reading}

Byrne, L., Stratford, A. and Davidson, L. (2018), "Speaking out: the global need for lived experience leadership", Psychiatric Rehabilitation Journal, Vol. 41 No. 1, pp. 76-79.

Corrigan, P.W., Larson, J.E. and Rosch, N. (2009), "Self-stigma and the "why try' effect: impact on life goals and evidence-based practices”, World Psychiatry, Vol. 8, pp. 75-81.

\section{Corresponding author}

Darrell Norman Burrell can be contacted at: dburrel12@thechicagoschool.edu

For instructions on how to order reprints of this article, please visit our website: www.emeraldgrouppublishing.com/licensing/reprints.htm Or contact us for further details: permissions@emeraldinsight.com 